\title{
An Aircraft Wake Turbulence Model Based on Lidar Detection
}

\author{
Mingyu Jiang, Qi Wang, Zhuo Zhang, Zichen Nan and Zhiyuan Shen + \\ College of Civil Aviation, Nanjing University of Aeronautics and Astronautics, Nanjing, China, 210016
}

\begin{abstract}
With the development of civil aviation, the influence of wake turbulence on aircraft flight safety and airport air traffic control is increasingly prominent. In China, the current wake interval standard based on traditional theoretical analysis and numerical simulation is not accurate enough, which further decreases the efficient operation of the airport. This paper first introduces the mechanism of aircraft wake turbulence. Secondly, a wake turbulence model based on lidar detection method is established. Thirdly, the advantages and disadvantages of the proposed method are analyzed compared with the state-of-the-art methods. The research results of this paper provide a theoretical basis for the wake reduction standard of Chinese civil aviation.
\end{abstract}

Keywords: aircraft, wake turbulence, lidar, wake turbulence separation

\section{Introduction}

As the by-product of aircraft lift, aircraft wake turbulence has the characteristics of strong, stable and large space scale. In the stages of cruise and approach, due to its long retention time, when the rear aircraft enters the wake area of the front aircraft, the roll torque induced by its strong vortex structure will cause the fuselage of the rear aircraft to shake, sink and change the flight state, resulting in flight accidents.

According to the characteristics of changes in the velocity of wake turbulence, trailing vortex velocity of the traditional description model mainly includes Rankine vortex model, Lamb-Oseen vortex model, HallockBurnham vortex model, Adapted vortex model and Multiple scale vortex model, etc. GERZ(2002), KOPP(1994), PROCTOR(1998) et al. [1] verified the standard trailing vortex tangential velocity model of Hallock-Burnham vortex, suggesting that the model can accurately describe the tangential velocity change of aircraft trailing vortex after ground effect.

At present, a number of aircraft wake turbulence detection experiments have been carried by using lidar. In the early days, the lidar commonly used was continuous wave laser radar, and the representative one was the wake observation carried out by Hallock et al. [2] at Heathrow airport. However, due to the limited performance of the CW laser radar, the observation distance can only reach a few hundred meters, so it is easy to fail to capture the wake motion or the wake positioning. In the early 21 st century, with the emergence of pulse lidar with better performance and stability, the adaptability, observation accuracy and accuracy of aircraft wake observation environment have been further improved. Friedrich [3-4] et al. verified for the first time the feasibility of $2 \mu \mathrm{m}$ Doppler radar to detect aircraft wake at a distance (covering a range of more than $1 \mathrm{~km}$ ), and proposed a fourth-order data processing algorithm to invert wake ring amount based on radar observation results.

In order to improve the wake turbulence separation standard and improve the runway capacity on the side runway, the foundation is laid for the subsequent use of lidar in wake detection. This paper will elaborate the generation principle of wake vortex, HB wake vortex model and lidar detection principle, and analyze the lidar detection model. Finally, summarized the advantages and disadvantages of lidar and its detection methods, and proposed corresponding solutions.

\footnotetext{
${ }^{+}$Corresponding author.

E-mail address: shenzy@nuaa.edu.cn
} 


\section{Wake Turbulence Generation Mechanism and HB Wake Model}

\subsection{Mechanism}

Wake turbulence is caused by the lift of the aircraft. The aerodynamic shows when the wings generate lift, under the wing of the pressure is higher than on the wings of the pressure. The air flow at tip of the wing rolls up from below and has the relatively backward flow velocity. The result is behind the two wings form two spiral that rotating flow backwards. The rotation of the two vortexes in the opposite direction, therefore is called the wingtip wake turbulence [5]. Due to the mutual inducement of vortexes and the reason of gravity, the vortexes, while spinning and spreading, fall at a certain speed.

\subsection{HB Wake Turbulence Model}

There is a pressure difference between the upper and lower wing surfaces of the aircraft, and the air in the passing area is disturbed and finally presents as a pair of vortexes, whose strength is measured by the attachment vorticity. The attachment vorticity is proportional to the weight of the aircraft and inversely proportional to the speed of the aircraft. The initial attachment vorticity is expressed as follows [6]:

$$
\mathrm{T}_{0}=\frac{M \mathrm{~g}}{\rho \mathrm{s} B V}=\frac{M \mathrm{~g}}{\rho \mathrm{b}_{0} \mathbf{V}}
$$

M,B represent the mass and wingspan of the aircraft, $G$ is the gravitational acceleration, $\rho$ is the density of the surrounding air, $\mathrm{S}$ is the load coefficient, $\mathrm{V}$ is the velocity of the aircraft, and $\mathrm{b}_{0}$ is the initial vortex core spacing.

At present, the commonly used wake vortex model is Hallock Burnham tangential velocity model, whose expression is concise and relatively close to the actual situation. The HB model is expressed as follows [6]:

$$
\boldsymbol{\nu}_{T}(\mathbf{r})=\frac{T_{0}}{2 \pi \mathbf{r}} \frac{\boldsymbol{r}^{2}}{\boldsymbol{r}^{2}+\boldsymbol{r}_{c}^{2}}=\frac{\boldsymbol{T} \mathrm{o}}{2 \pi\left(\boldsymbol{r}^{2}+\boldsymbol{r}_{c}^{2}\right)}
$$

$\mathrm{T}_{0}$ is the initial vortex ring quantity, $\mathrm{r}$ is the distance from the vortex core, $\mathrm{c}_{\mathrm{r}}$ is the radius of the vortex core (usually set as $0.052 \mathrm{~b} 0$ ), and $\mathrm{v}_{\mathrm{T}}(\mathrm{r})$ is the tangential velocity.

When the wing-tip wake turbulence forms, it will produce its induced velocity field and drift downward at the same time. During the formation of wake turbulence, the overall velocity field is synthesized by these two parts. The downward drift velocity can be expressed as [7]:

$$
\omega_{0}=\frac{T_{0}}{2 \pi b_{0}}=\frac{2 T_{0}}{\pi^{2} B}
$$

According to formula (3), in a certain flight environment or state, the larger the mass of the aircraft, the stronger the generated wake turbulence $\mathrm{x}$, the slower the attenuation, and the faster the downward velocity. Therefore, the wake turbulence strength of heavy aircraft is larger than that of medium aircraft, while that of medium aircraft is much stronger than that of light aircraft. In addition, the wake turbulence caused by the same aircraft is stronger at low speed than at high speed.

\section{Principle and Detection Method of Lidar}

\subsection{Lidar Composition}

The basic composition of lidar (as shown in Figure 1) includes three parts: transmitting, receiving and post signal processing, and the mechanism to coordinate the three parts. The transmitting part includes a laser transmitter, a laser modulator, a beam controller, and an optical transmitting antenna. The receiving part includes an optical receiving antenna photodetector. 


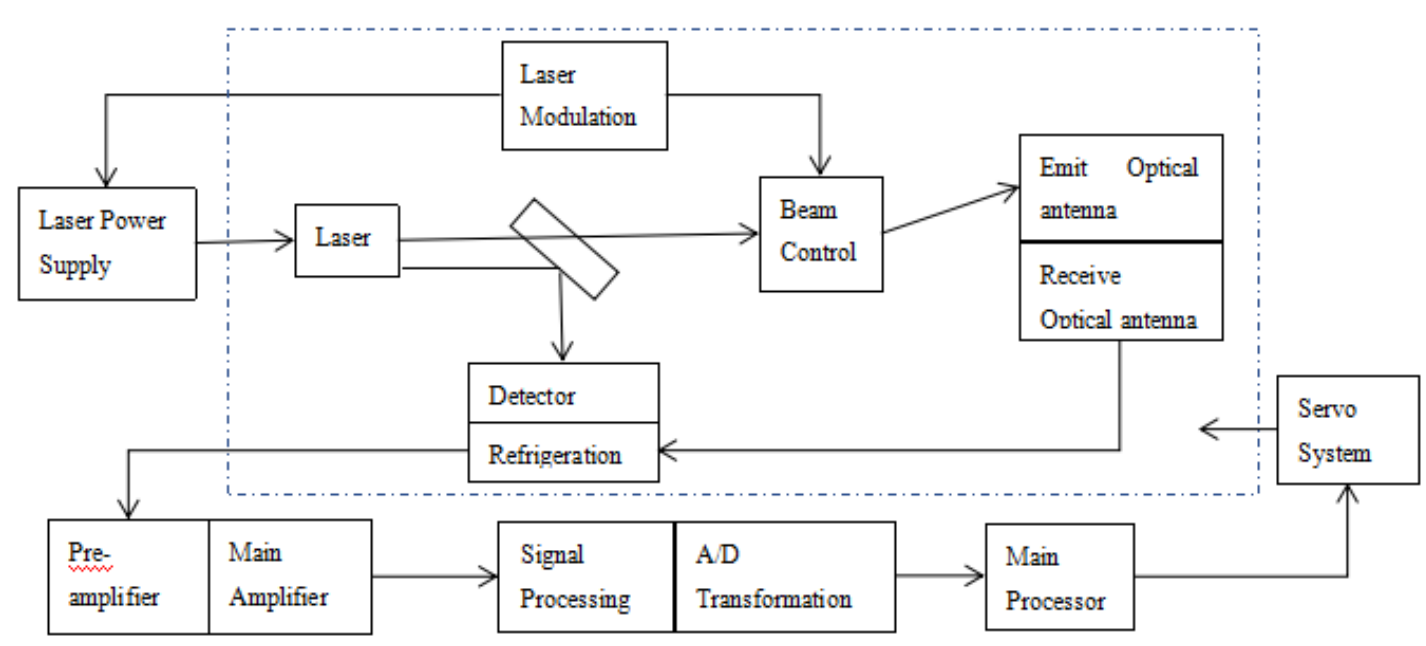

Fig. 1: Basic block diagram of typical laser radar

\subsection{Principle of Lidar Detection}

Lidar mainly emits laser beam to scan a specific airspace, and receives the back scattering signal of laser from atmospheric molecules or aerosol particles through a telescope. In the detection airspace, due to the thermal motion of atmospheric molecules and the Brownian motion of aerosol particles, the Doppler broadening of backscattered signals is generated, while the monolithic average velocity of particles results in the Doppler shift of atmospheric echo signals. The Doppler shift of the atmospheric echo signal at different points is detected by the coherent method, and then the radial velocity of the atmospheric molecules or aerosol particles along the laser beam at this point is inversed [7]. The system uses the power amplifier structure of the main control oscillator, uses a single laser as the frequency reference oscillator, and its output is generating frequency shift and emoting later by the acousto-optic adjustment devices. At the same time, a small part of the power extracted directly from the laser as the local oscillator signal through the fiber coupler. The light beam emitted by the optical antenna is backscattered through the air molecules and aerosols in the wake vortex of the aircraft and received by the optical antenna again. After filtering by a narrow-band filter, the echo signal is mixed with the local-oscillator light which reaches the detector through long fiber in the Balanced detector, then, it enters the data acquisition system after being amplified by intermediate frequency amplifier. The collected data are subsequently processed to obtain the information of the wake vortex in the end.

If the radial velocity of atmospheric molecules or aerosol particles along the laser beam is set to $V_{R}$, after scattering, a single frequency laser with the original frequency of $f_{0}$ will come up a Doppler frequency shift $\triangle$ $f_{D}$, there is the following relationship between $\triangle f_{D}$ and $V_{R}[8]$.

$$
\triangle f_{D}=2 f_{0} \frac{v_{R}}{c}=\frac{2}{\lambda_{0}} v_{R}
$$

formula: $\mathrm{C}$ is the speed of light; lambda $\lambda_{0}$ is the laser wavelength corresponding to the frequency $\mathrm{f}_{0}$.

Generally, coherent means can be used to measure Doppler frequency shift, because compared with incoherent detection, it not only has higher detection sensitivity and accuracy, but also can achieve some functions that incoherent detection technology can not achieve. According to formula (4), the instantaneous velocity of any detection target point relative to the detection light source can be calculated. By integrating the velocity curve, the relative position of each target point in the scanning time can be obtained, and the distance corresponding to the target at different detection times can be displayed. The radial velocity is calculated according to the reciprocal of the relative time, and the value of velocity directly corresponds to the change of Doppler frequency of echo signal. In the Doppler scanning detection, it only needs to demodulate, reshape, subdivide and count the signal to extract the position information of the target completely[9].

\subsection{RHI Scanning Mode}

When Doppler lidar uses to measure the velocity of atmospheric molecules or aerosol particles, only the velocity component consistent with the same propagation direction of the laser beam can cause the doppler frequency shift of the backscatter light signal, so the laser transverse detection method is used to detect the wake vortex of aircraft, which is often called RHI scanning method.[7] 
The forward of $\mathrm{X}$ axis is defined as the datum direction, and the aircraft takes off along the direction; $\mathrm{Z}$ axis is the longitudinal section perpendicular to the datum direction, and $\mathrm{Y}$ axis is the transverse section perpendicular to the datum direction. The YOZ plane and the plane parallel to it constitute the section of the vortex section of the aircraft at different distances.

On account of the influence of aircraft wake vortex is air disturbance when the aircraft flew over vertical plane, the laser beam vertical sweep trailing vortex, which will get trailing vortex speed transient plane distribution, the scanning principle as shown in Fig. 2.

Under the ideal condition, the magnitudes of the tangential velocity modes at the points on the wake vortex ring are equal, during the process of scanning, trailing vortex tangential velocity $\mathrm{v}(\mathrm{r})$ and radar radial velocity $\mathrm{v}(\mathrm{r})$ will form a scanning angle, so that the tangential velocity of trailing vortex will be projected to the scanning direction of radar beam, the radar echo of the trailing vortex radial velocity is formed, which is specifically expressed as: According to change trend of the standard model of the radial velocity of the trailing vortex, the positive-negative velocity of the left and right trailing vortex changes centres on the vortex core which is the center of symmetry. The speed gradually close to zero at infinity due to the influence of the air resistance. When $r$ approaching the vortex core, the radial velocity values of the left and right vortexes increase with the decrease of the scanning angle $\delta$ gradually and reach a maximum near the vortex core [11]. Afterwards, after the vortex core point, there is a sudden speed change. When the lidar detection in the RHI mode, azimuth angle is fixed at the time of measurement, change the pitching angle of laser radar receiving echo by fast Fourier transform to get radial wind speed with a fixed distance from the door. When it comes to velocity, a negative value approaching the direction of the lidar and a positive value away from the lidar, the numerical value in this direction is the value of the speed. When the radar sweeps a circle in the set scan pitch angle, we will get a radial velocity wind field with a slice:

$$
V_{R}=\left\{v_{R}\left(\rho_{i}, \theta_{j}\right)\right\}, \quad i \in[1, n], \quad j \in[1, m]
$$

Thereinto, $\mathrm{n}$ and $\mathrm{m}$ are respectively the number of discrete values of pitch Angle obtained by lidar detection scanning and the number of radial range gate. The number of distance gate of the lidar used in field detection $\mathrm{n}=56$.

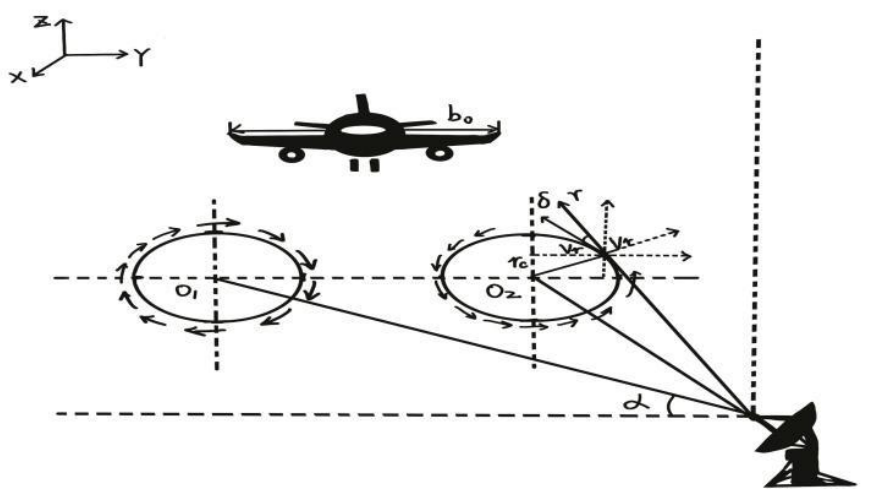

Fig. 2: Schematic diagram of wake vortex measurement of laser radar

RHI scanning method can provide the detailed information of the vertical structure of cloud and rain echoes intuitively. Since the laser beam emitted by this scanning method is basically in the wake vortex cross-sectional plane when the wake vortex is detected, only can the radial component of the vortex velocity along the laser beam at each point on the wake vortex profile be obtained. Therefore, as long as the tangential velocity of the wake vortex can be extracted from the radial velocity obtained by laser detection. The wake vortex parameter information such as vortex core location, vortex core radius and vortex circulation can be obtained based on the wake vortex parameter estimation algorithm. In total, under the RHI scanning method, if the radial velocity of each point obtained from the laser beam at different elevation angles is compared, it takes out the maximum value and performs curve fitting on the above-mentioned radial velocity debt series, so that the tangential velocity distribution curve of the wake vortex profile can be obtained [7]. 


\section{Comparative Analysis of Awake Detection Methods}

The advantages and disadvantages of continuous-wave laser radar and pulsed liadar based on the differences of laser emission waveform classification as shown in Table 1.

Table 1: Classification by laser emission waveform

\begin{tabular}{cll}
\hline & \multicolumn{1}{c}{ Advantage } & \multicolumn{1}{c}{ Disadvantage } \\
\hline & $\begin{array}{l}\text { The detection range is wide and can cover } \\
\text { the whole world; }\end{array}$ & $\begin{array}{l}\text { The observation distance is } \\
\text { short, and it is easy to fail to } \\
\text { Continuous-wave } \\
\text { laser radar }\end{array}$ \\
$\begin{array}{l}\text { High spatial resolution, high temporal } \\
\text { resolution, and high measurement accuracy; } \\
\text { Molecular scattering measurements across } \\
\text { the globe. }\end{array}$
\end{tabular}

The detection range has been increased, and Pulsed has higher measurement accuracy, high lidar spatial resolution and high temporal resolution.

During lidar detection, the scattering of suspended particles will be absorbed, and the combined effect on atmospheric attenuation will affect the coherence of the light source, which will reduce the quality of data detected and transmitted, suitable for working in sunny days.

Table 2 compares the advantages and disadvantages of different radar equipment, including Lidar, $\mathrm{X}$-band Radar, and Millimeter-wave radar.

Table 2: Comparison of various radar equipment

\begin{tabular}{|c|c|c|}
\hline & Advantage & Disadvantage \\
\hline & $\begin{array}{c}\text { Wide detection range; } \\
\text { High measurement accuracy; }\end{array}$ & $\begin{array}{c}\text { Fast attenuation of optical signal } \\
\text { power; }\end{array}$ \\
\hline Lidar & $\begin{array}{l}\text { High spatial resolution; } \\
\text { High time resolution. }\end{array}$ & $\begin{array}{c}\text { The coherence of light source is } \\
\text { easy to be affected; } \\
\text { Severe weather disturbance }\end{array}$ \\
\hline $\begin{array}{l}\text { X-band } \\
\text { radar }\end{array}$ & $\begin{array}{c}\text { Under extreme weather conditions such as } \\
\text { rain and fog, the detection of wake is more } \\
\text { sensitive. }\end{array}$ & $\begin{array}{l}\text { Complex operation; } \\
\text { High cost; }\end{array}$ \\
\hline $\begin{array}{l}\text { Millimeter-wave } \\
\text { radar }\end{array}$ & $\begin{array}{c}\text { Doppler characteristics of wake in fog and } \\
\text { cloud under resolution and observation } \\
\text { perspective. }\end{array}$ & $\begin{array}{c}\text { Expensive devices; } \\
\text { Low power of transmitter; } \\
\text { Large loss of waveguide device. }\end{array}$ \\
\hline
\end{tabular}

Technically speaking, the traditional lidar technology is very mature, while the solid-state lidar and hybrid solid-state lidar are still in the initial stage. For wake detection in complex weather conditions, X-band radar and millimeter wave radar can be used for complementary observation of lidar.

\section{Conclusion}

This paper analyzes the mechanism of aircraft wake generation. Based on the basic principle of lidar detection of wake, by analyzing the parameters of HB wake model, a lidar-based wake detection model was established. Compared with other wake detection methods, the advantages and disadvantages of lidar's own detection method and accuracy are compared and analyzed, and its application in RHI scanning mode is analyzed. The research results in this paper can provide a certain theoretical basis for the reduction of wake standards in China's civil aviation, and provide a favorable guarantee for the safe operation and efficiency improvement of civil aviation. In the subsequent research, we will continue to improve the existing radar 
detection model, add the characteristic parameters of actual operation, further enrich and develop the wake vortex detection theory, and apply the research results to the lateral runway.

\section{Acknowledgement}

The authors would like to thank the financial support by the Nanjing University of Aeronautics and Astronautics for the Research on the lateral runway wake monitoring and runway capacity lifting based on lidar Program（No.2020CX00751）.

\section{References}

[1] FREHLICH R, SHARMAN R. Maximum likelihood estimates of vortex parameters from simulated coherent doppler lidar data Journal of Atmospheric\& Oceanic Technology, 2005, 22(22): 117-130.

[2] HALLOCKJ, BURNHAM D, HALLOCK J, et ai. Decay characteristics of wake vortices from jet transport aircraft [J]. Journal of aircraft, 2013, 50(1): 82-87.

[3] VAUGHANJM, HARRISM. Lidar measurement of b747 wakes: observation of a vortex within a vortex[J]. Aerospace Science \&Technology, 2001, 5(6): 409-411.

[4] KEPPF, RAHMS, SMALIKHO. Characterization of aircraft wake vortices by $2-\mu \mathrm{m}$ pulsed doppler lidar [J]. Journal of Atmospheric \& Oceanic Technology, 2004, 21(2):194-206.

[5] Xing Suxia, Zhang Junju, Chang Benkang, et al. Recent development and status of uncooled IR thermal imaging technology [J]. Infrared and Laser Engineering, 2004, 33 (5): 441-444. (in Chinese)

[6] MURPHY B, O'CALLAGHAN J, FOX M, et al. Overview of the Structures Investigation for the American Airlines Flight 587 Investigation[C]/46th AIAA/ASME/ASCE/AHS/ASC Structures, Structural Dynamics and Materials Conference. Washington DC, USA: 2013:3369-3373.

[7] HUYH, WUYH. Research on aircraft evolution and the Lidar measurement technology[J]. Infrared and Laser Engineer in, 2011,40(6):1063-1069. (in Chinese).

[8] Kopp F, Rahm S, Smalikho I. Characterization of aircraft wake vortices by $2-\mu \mathrm{m}$ pulsed Doppler lidar [J]. Journal of Atmospheric and Oceanic Technology,2004,21(2):194-206.

[9] Zhang Xiaoyuan, Hong Xin, He Shunzhong, etal. Study on measurement technique of scattering-displacement utilizing laser doppler effect [J] Chinese Journal of Lasers,2000,27(8):751-755. (in Chinese).

[10] WU Y H, HU Y H, DAI D Ch, et al. Research on the technique of aircraft wake vortex detection based on 1. 5um Doppler lidar] Acta Photonica Sinica, 2011, 40(6): 811-817 (in Chinese). 